\title{
A unifying approach to fractional Lévy processes
}

Sebastian Engelke and Jeannette H.C. Woerner

Preprint 2010-16

Dezember 2010

Fakultät für Mathematik

Technische Universität Dortmund

Vogelpothsweg 87

44227 Dortmund

$\underline{\text { tu-dortmund.de/MathPreprints }}$ 



\title{
A unifying approach to fractional Lévy processes
}

\author{
Sebastian Engelke*and Jeannette H.C. Woerner ${ }^{\dagger}$ \\ University of Göttingen and Technical University of Dortmund
}

\begin{abstract}
Starting from the moving average representation of fractional Brownian motion fractional Lévy processes have been constructed by keeping the same moving average kernel and replacing the Brownian motion by a pure jump Lévy process with finite second moments. Another way was to replace the Brownian motion by an alpha-stable Lévy process and the exponent in the kernel by $H-1 / \alpha$. We now provide a unifying approach taking kernels of the form $a\left((t-s)_{+}^{\gamma}-(-s)_{+}^{\gamma}\right)+b\left((t-s)_{-}^{\gamma}-(-s)_{-}^{\gamma}\right)$, where $\gamma$ can be chosen according to the existing moments and the Blumenthal-Getoor index of the underlying Lévy process. These processes may exhibit both long and short range dependence. In addition we will examine further properties of the processes, e.g. regularity of the sample paths and the semimartingale property.
\end{abstract}

key words: fractional Lévy process, linear fractional stable motion, fractional Brownian motion, semimartingale, correlation, long-range dependence, Blumenthal-Getoor index

MSC 2010: 60G22, 60E07

\section{Introduction}

Fractional Brownian motion provides a classical approach for modelling dependence structures, both allowing for long range dependence, as e.g. often seen in financial data, or short range dependence, as e.g. in turbulence data. However, fractional Brownian motion is a zero mean Gaussian process and hence possesses normally distributed increments, which in many circumstances is not a realistic feature for modelling. This leads to the idea to merge the desirable properties of the correlation structure of fractional Brownian motion with those of infinitely divisible distributions of Lévy processes resulting in fractional Lévy processes.

One way of defining fractional Brownian motion with Hurst parameter $H$ is via the moving average representation (cf. Mandelbrot and Van Ness (1968))

$$
B_{t}^{H}=C \int\left(a\left((t-s)_{+}^{H-1 / 2}-(-s)_{+}^{H-1 / 2}\right)+b\left((t-s)_{-}^{H-1 / 2}-(-s)_{-}^{H-1 / 2}\right)\right) d B_{s},
$$

where $x_{+}=\max (x, 0), x_{-}=\max (-x, 0), a, b \in \mathbb{R}, H \in(0,1), C$ a normalizing constant and $\left(B_{t}\right)_{t \in \mathbb{R}}$ denotes a standard two-sided Brownian motion. The resulting processes possess a correlation of the form

$$
E\left(B^{H}(t) B^{H}(s)\right)=\frac{1}{2}\left(|t|^{2 H}+|s|^{2 H}-|t-s|^{2 H}\right) .
$$

\footnotetext{
*Institut für Mathematische Stochastik, Georg-August-Universität Göttingen, Goldschmidtstraße 7, D-37077 Göttingen, Germany, email: sengelk@uni-goettingen.de

${ }^{\dagger}$ Fakultät für Mathematik, Technische Universität Dortmund, Vogelpothsweg 87, D-44227 Dortmund, Germany. email: jeannette.woerner@math.tu-dortmund.de
} 
Hence for $H \in(0.5,1)$ the process has positively correlated increments and exhibits long range dependence, whereas for $H \in(0,0.5)$ it has negatively correlated increments and exhibits short range dependence. For $H=0.5$ the process coincides with the standard Brownian motion. Furthermore, fractional Brownian motions do not belong to the class of semimartingales except for $H=0.5$ and they possess Hölder continuous sample paths of order less than $H$.

Benassi et al. (2004) and Marquardt (2006) introduced fractional Lévy processes by replacing the Brownian motion in the moving average representation by a pure jump Lévy process without drift, which possesses finite second moments. Especially Marquardt (2006) was mainly interested in the case of long range dependence.

A different approach of generalizing fractional Brownian motion in the direction of Lévy processes was followed by Samorodnitsky and Taqqu (1994). They replaced the Brownian motion in the moving average representation by an $\alpha$-stable process with $\alpha \in(0,2)$ and the exponent in the kernel by $H-1 / \alpha$ leading to linear fractional stable motions. Due to the self-similarity of stable processes also linear fractional stable motion keeps this property of fractional Brownian motion, whereas general fractional Lévy processes are not self-similar.

Based on the special form of the kernel function fractional Lévy processes have the same correlation structure as fractional Brownian motion, but other properties change. Looking at the path behaviour of fractional Lévy processes, they are only Hölder continuous in the long range dependent case but with a different Hölder exponent, whereas for the short range dependent case the sample paths are unbounded, leading to peaks which might be interesting for modelling e.g. electricity data. Furthermore, other than fractional Brownian motion fractional Lévy processes might lead to semimartingales of finite variation in the long range dependent case, $\mathrm{cf}$. Basse and Pedersen (2009) and Bender et al. (2010).

In this paper we now provide a unifying approach to fractional Lévy processes. We derive sufficient conditions on the exponent of the kernel function leading to a larger class of processes, especially for the short range dependent case. We will see that the upper bound of the exponent depends on the existing moment of the underlying Lévy process and the lower bound on the Blumenthal-Getoor index, i.e. the jump activity. In some circumstances only an appropriate choice of the drift component in the Lévy process ensures the existence of fractional Lévy processes. In addition we provide both distributional and path properties of the constructed processes, e.g. regularity of the sample paths and semimartingale property, and compare them to fractional Brownian motion. Especially we see that for fractional Brownian motion and fractional Lévy processes the characteristic quantities, i.e. exponent of the kernel function, exponent in the correlation function, maximal Hölder exponent and self-similarity index do not stay in the same functional relationship. While for fractional Brownian motion one parameter $H$ is sufficient to describe them all, for fractional Lévy processes in general we need three parameters, the exponent of the kernel function, the Blumenthal-Getoor index and the maximal existing moment, if it is less than two.

\section{Integrability conditions and definition of fractional Lévy processes}

Let $\left(L_{t}\right)_{t \geq 0}$ be a Lévy process without Brownian component and characteristic triplet given by $(b, 0, \nu)$, where $\nu$ is the Lévy measure and $b \in \mathbb{R}$ the drift of $L$. More precisely, for $t \geq 0$, the characteristic function of $L$ is given by $E\left[e^{\mathrm{i} u L(t)}\right]=e^{t \psi(u)}$ for all $u \in \mathbb{R}$, where

$$
\psi(u)=\mathrm{i} u b+\int_{\mathbb{R}_{0}}\left(e^{\mathrm{i} u x}-1-\mathrm{i} u x 1_{\{|x| \leq 1\}}\right) \nu(d x) .
$$


From now on we will always work with a two-sided version $\left(L_{t}\right)_{t \in \mathbb{R}}$ of the above processes, i.e. $L(t)=L^{1}(t)$ if $t \geq 0$ and $L(t)=-L^{2}\left(-t_{-}\right)$if $t<0$, where $L^{1}$ and $L^{2}$ denote independent copies of $L$. We consider integrals of deterministic functions with respect to these Lévy processes. Defining

$$
\Lambda((a, b])=L(b)-L(a), \quad a, b \in \mathbb{R} ; a<b
$$

yields an independently scattered, infinitely divisible random measure on $\mathbb{R}$. Rajput and Rosiński (1989) developed and integration theory for this kind of random measures. In this framework a measurable function $f$ is called $\Lambda$-integrable (or $L$-integrable) if it is the almost sure limit of approximating simple functions whose integrals converge in probability. The limit of these integrals is defined as the $L$-integral of $f$. In this case it is independent of the approximating sequence of functions (see Urbanik and Woyczynski (1967) for details). Throughout this paper the integrals will be understood in this sense.

Let $(\Omega, \mathcal{A}, \mathbb{P})$ be the underlying probability space and let $L_{0}(\mathbb{R})$ denote the space of measurable functions on $\mathbb{R}$. According to Rajput and Rosiński (1989, Theorem 2.7), $f \in L_{0}(\mathbb{R})$ is $L$-integrable if and only if the following two conditions hold:

$$
\begin{aligned}
& \int_{\mathbb{R}} \int_{\mathbb{R}_{0}}\left(|f(s) x|^{2} \wedge 1\right) \nu(d x) d s<\infty, \\
& \int_{\mathbb{R}}\left|f(s)\left(b+\int_{\mathbb{R}_{0}} x\left(1_{\{|f(s) x| \leq 1\}}-1_{\{|x| \leq 1\}}\right) \nu(d x)\right)\right| d s<\infty .
\end{aligned}
$$

Furthermore, for $p \in \mathbb{R}_{+}$the so-called Musielak-Orlicz space is defined as

$$
L_{\phi_{p}}(\mathbb{R})=\left\{f \in L_{0}(\mathbb{R}) \text { is } L \text {-integrable and } \int_{\mathbb{R}} \int_{\mathbb{R}_{0}} 1_{\{|f(s) x|>1\}}|f(s) x|^{p} \nu(d x) d s<\infty\right\} .
$$

Note that $L_{\phi_{p}}(\mathbb{R})$ is a complete linear metric space (for details see Musielak (1983)). Applying Theorem 3.3 of Rajput and Rosiński (1989) to our setting yields the following proposition.

Proposition 1. Let $q \in \mathbb{R}_{+}$and $0 \leq p \leq q$. Suppose that for any $t \in \mathbb{R}$ we have $E|L(t)|^{q}<\infty$. Then

$$
\left\{f \in L_{0}(\mathbb{R}): f \text { is L-integrable and } E\left|\int_{\mathbb{R}} f(s) L(d s)\right|^{p}<\infty\right\}=L_{\phi_{p}}(\mathbb{R})
$$

and the linear mapping

$$
L_{\phi_{p}}(\mathbb{R}) \ni f \mapsto \int_{\mathbb{R}} f(s) L(d s) \in L^{p}(\Omega, \mathbb{P})
$$

is continuous.

For $\gamma \in \mathbb{R}$ let us define the following functions:

$$
\begin{aligned}
f_{\gamma}^{+}(t, s) & =(t-s)_{+}^{\gamma}-(-s)_{+}^{\gamma}, \\
f_{\gamma}^{-}(t, s) & =(t-s)_{-}^{\gamma}-(-s)_{-}^{\gamma}, \\
f_{\gamma}(t, s) & =|t-s|^{\gamma}-|s|^{\gamma}=f_{\gamma}^{+}(t, s)-f_{\gamma}^{-}(t, s) .
\end{aligned}
$$

At first, we only consider the function $f_{\gamma}^{+}$. For two functions $h$ and $g$ on $\mathbb{R}$ we will frequently use the notation $f(s) \sim g(s)$ as $s \rightarrow a \in[-\infty, \infty]$ in the sense of $\lim _{s \rightarrow a} f(s) / g(s)=C \in \mathbb{R}$.

Proposition 2. For $f_{\gamma}^{+}$as above, $\gamma<1$ and $t \in \mathbb{R}$ fixed we have the following.

$$
\text { 1. }\left|f_{\gamma}^{+}(t, s)\right| \sim|s|^{\gamma-1} \text { for } s \rightarrow-\infty \text {. }
$$


2. (a) If $\gamma>0$ then $\left|f_{\gamma}^{+}(t, \cdot)\right|$ is bounded on $\mathbb{R}$.

(b) If $\gamma<0$ then $\left|f_{\gamma}^{+}(t, s)\right| \sim|s|^{\gamma} \rightarrow \infty$ for $s \nearrow 0$ and $\left|f_{\gamma}^{+}(t, s)\right| \sim|t-s|^{\gamma} \rightarrow \infty$ for $s \nearrow t$.

3. Let $\delta>0$.

(a) $\left|f_{\gamma}^{+}(t, \cdot)\right|^{\delta}$ is integrable at $-\infty$ iff $\delta>\frac{1}{1-\gamma}$.

(b) $\left|f_{\gamma}^{+}(t, \cdot)\right|^{\delta}$ is integrable at 0 and $t$ iff either $\gamma>0$ or $\gamma<0$ and $\delta<\frac{1}{-\gamma}$.

Proof. 1. Using l'Hôpital's rule we obtain

$$
\begin{aligned}
\lim _{s \rightarrow-\infty} \frac{\left|f_{\gamma}^{+}(t, s)\right|}{|s|^{\gamma-1}} & =\lim _{s \rightarrow-\infty} \frac{\left|\left(1-\frac{t}{s}\right)^{\gamma}-1\right|}{|s|^{-1}} \\
& =\lim _{u \searrow 0} \frac{\left|(1+u t)^{\gamma}-1\right|}{u} \\
& =\lim _{u \searrow 0} \frac{\left|\gamma(1+u t)^{\gamma-1} t\right|}{1}=|\gamma t| .
\end{aligned}
$$

2. Obvious from the definition of $f_{\gamma}^{+}$.

3. (a) Let $N<\min (0, t)$, then we observe that by the first part of this proposition $\int_{-\infty}^{N}\left|f_{\gamma}^{+}(t, s)\right|^{\delta} d s<\infty$ is equivalent to $\int_{-\infty}^{N}|s|^{\delta(\gamma-1)} d s<\infty$ which holds if and only if $\delta>\frac{1}{1-\gamma}$.

(b) If $\gamma>0$ the assertion follows from the boundedness of $f_{\gamma}^{+}$. Let now $0<\epsilon<|t|$. By part 2(b) we note that $\int_{-\epsilon}^{0}\left|f_{\gamma}^{+}(t, s)\right|^{\delta} d s<\infty$ if and only if $\int_{-\epsilon}^{0}|s|^{\delta \gamma} d s<\infty$. The latter is clearly equivalent to $\delta<\frac{1}{-\gamma}$.

We are now in a position to state certain integrability conditions of $f_{\gamma}^{+}$depending on $\gamma$ and the Lévy measure $\nu$.

Proposition 3. Let $A_{1}=\left\{(x, s) \in \mathbb{R}_{0} \times \mathbb{R}:\left|f_{\gamma}^{+}(t, s) x\right|>1\right\}$ and $\gamma<1$.

1. For $\delta \geq 0$ we have that

$$
\int_{A_{1}}\left|f_{\gamma}^{+}(t, s) x\right|^{\delta} \lambda \otimes \nu(d s d x)<\infty
$$

is equivalent to the following:

(i) $\int_{|x|>1}|x|^{\delta \vee \frac{1}{1-\gamma}} \nu(d x)<\infty$,

(ii) $\delta<-1 / \gamma($ for $\gamma<0)$,

(iii) $\int_{|x|<1}|x|^{-1 / \gamma} \nu(d x)<\infty($ for $\gamma<0)$.

2. For $\xi>0$

$$
\int_{A_{1}^{c}}\left|f_{\gamma}^{+}(t, s) x\right|^{\xi} \lambda \otimes \nu(d s d x)<\infty
$$

holds if and only if 

(i) $\xi>\frac{1}{1-\gamma}$,
(ii) $\int_{|x|>1}|x|^{\frac{1}{1-\gamma}} \nu(d x)<\infty$,
(iii) $\int_{|x|<1}|x|^{\xi} \nu(d x)<\infty$,
(iv) $\int_{|x|<1}|x|^{-1 / \gamma} \nu(d x)<\infty($ for $\gamma<0)$.

Proof. 1. Applying Tonelli's Theorem, (4) is equivalent to

$$
\int_{\mathbb{R}_{0}}|x|^{\delta} \int_{\left\{s \in \mathbb{R}:\left|f_{\gamma}^{+}(t, s)\right|>\frac{1}{|x|}\right\}}\left|f_{\gamma}^{+}(t, s)\right|^{\delta} d s \nu(d x)<\infty .
$$

By Prop. 2.3 for any $x \in \mathbb{R}_{0}$ the inner integral is finite and continuous in $x$ if and only if either $\gamma>0$ or $\gamma<0$ and $\delta<\frac{1}{-\gamma}$. In this case we have to establish the convergence of the outer integral only for $|x| \rightarrow 0$ and $|x| \rightarrow \infty$.

For the latter case let $N$ be large enough, such that we can approximate $\left|f_{\gamma}^{+}(t, s)\right|$ by $|s|^{\gamma-1}$ for all $s<-N$ as in Prop. 2. We then clearly have for large $M$ that by Prop. 2.1

$$
\int_{|x|>M}|x|^{\delta} \int_{\left\{s \in \mathbb{R}:\left|f_{\gamma}^{+}(t, s)\right|>\frac{1}{|x|}\right\}}\left|f_{\gamma}^{+}(t, s)\right|^{\delta} d s \nu(d x)<\infty
$$

holds if and only if

$$
\begin{aligned}
& \int_{|x|>M}|x|^{\delta} \int_{\left\{s \in \mathbb{R}:|s|^{\gamma-1}>\frac{1}{|x|}\right\} \cap(-\infty,-N]}|s|^{\delta(\gamma-1)} d s \nu(d x) \\
& =\int_{|x|>M}|x|^{\delta}\left(K_{1}+\int_{-|x|^{\frac{1}{1-\gamma}}}^{-N}|s|^{\delta(\gamma-1)} d s\right) \nu(d x) \\
& =\int_{|x|>M}|x|^{\delta}\left(K_{1}+K_{2}|x|^{-\delta+\frac{1}{1-\gamma}}\right) \nu(d x)<\infty,
\end{aligned}
$$

where $K_{1}, K_{2}$ denote constants. The last inequality is equivalent to

$$
\int_{|x|>1}|x|^{\delta \vee \frac{1}{1-\gamma}} \nu(d x)<\infty .
$$

Let us now consider the case $|x| \rightarrow 0$.

If $\gamma>0$, the set $\left\{s \in \mathbb{R}:\left|f_{\gamma}^{+}(t, s)\right|>\frac{1}{|x|}\right\}$ will be empty for sufficiently small $x$ which implies the convergence.

Now, let $\gamma<0$. Choose $\epsilon>0$ small enough such that for all $|x|<\epsilon$ the set $\{s \in \mathbb{R}$ : $\left.\left|f_{\gamma}^{+}(t, s)\right|>\frac{1}{|x|}\right\}$ is contained in $\left\{s \in \mathbb{R}:|s|^{\gamma}>\frac{1}{|x|}\right\} \cup\left\{s \in \mathbb{R}:|t-s|^{\gamma}>\frac{1}{|x|}\right\}$ and $\left|f_{\gamma}^{+}(t, s)\right|$ behaves as $|s|^{\gamma}$ on this set. Then we have that

$$
\int_{|x|<\epsilon}|x|^{\delta} \int_{\left\{s \in \mathbb{R}:\left|f_{\gamma}^{+}(t, s)\right|>\frac{1}{|x|}\right\}}\left|f_{\gamma}^{+}(t, s)\right|^{\delta} d s \nu(d x)<\infty
$$

by Prop. 2.2 is equivalent to

$$
\begin{aligned}
\int_{|x|<\epsilon}|x|^{\delta} \int_{\left\{s \in \mathbb{R}:|s|^{\gamma}>\frac{1}{|x|}\right\}}|s|^{\delta \gamma} d s \nu(d x) & =\int_{|x|<\epsilon}|x|^{\delta} K_{3}|x|^{-\delta-\frac{1}{\gamma}} \nu(d x) \\
& =K_{3} \int_{|x|<\epsilon}|x|^{-\frac{1}{\gamma}} \nu(d x)<\infty,
\end{aligned}
$$

where $\delta<-1 / \gamma$ and $K_{3}$ denotes a constant. 
2. Again by Tonelli's Theorem, (5) is equivalent to

$$
\int_{\mathbb{R}_{0}}|x|^{\xi} \int_{\left\{s \in \mathbb{R}:\left|f_{\gamma}^{+}(t, s)\right| \leq \frac{1}{|x|}\right\}}\left|f_{\gamma}^{+}(t, s)\right|^{\xi} d s \nu(d x)<\infty .
$$

By Prop. 2.3 for any $x \in \mathbb{R}_{0}$ the inner integral is finite and continuous in $x$ if and only if $\xi>\frac{1}{1-\gamma}$. In this case we have to establish the convergence of the outer integral only for $|x| \rightarrow 0$ and $|x| \rightarrow \infty$. Note that on the set $\left\{s \in \mathbb{R}:\left|f_{\gamma}^{+}(t, s)\right| \leq \frac{1}{|x|}\right\}$ we clearly have $\left|f_{\gamma}^{+}(t, s)\right|^{\xi} \leq|x|^{-\xi}$.

Now, consider $|x| \rightarrow \infty$ and choose $M$ large enough such that for all $|x|>M$ the set $\left\{s \in \mathbb{R}:\left|f_{\gamma}^{+}(t, s)\right| \leq \frac{1}{|x|}\right\}$ is contained $\left\{s \in \mathbb{R}:|s|^{\gamma-1} \leq \frac{1}{|x|}\right\} \cup[-N, N]=\{s \in \mathbb{R}:|s| \geq$ $\left.|x|^{\frac{1}{1-\gamma}}\right\} \cup[-N, N]$, for $N$ large enough. We then have by Prop. 2.1 that

$$
\int_{|x|>M}|x|^{\xi} \int_{\left\{s \in \mathbb{R}:\left|f_{\gamma}^{+}(t, s)\right| \leq \frac{1}{|x|}\right\}}\left|f_{\gamma}^{+}(t, s)\right|^{\xi} d s \nu(d x)<\infty
$$

holds if and only if

$$
\begin{aligned}
& \int_{|x|>M}|x|^{\xi}\left(\int_{-N}^{N}|x|^{-\xi} d s+\int_{-\infty}^{-|x|^{\frac{1}{1-\gamma}}}|s|^{\xi(\gamma-1)} d s\right) \nu(d x) \\
& =\int_{|x|>M}|x|^{\xi}\left(2 N|x|^{-\xi}+K_{1}|x|^{-\xi+\frac{1}{1-\gamma}}\right) \nu(d x)<\infty,
\end{aligned}
$$

where $K_{1}$ denotes a constant. The last inequality is equivalent to

$$
\int_{|x|>1}|x|^{\frac{1}{1-\gamma}} \nu(d x)<\infty .
$$

Let us now consider the case when $|x| \rightarrow 0$. First, assume that $\gamma>0$. For $\eta>0$ small enough we then observe that $\left\{s \in \mathbb{R}:\left|f_{\gamma}^{+}(t, s)\right| \leq \frac{1}{|x|}\right\}=\mathbb{R}$ for all $|x|<\eta$. Hence we obtain the equivalence of

$$
\int_{|x|<\eta}|x|^{\xi} \int_{\left\{s \in \mathbb{R}:\left|f_{\gamma}^{+}(t, s)\right| \leq \frac{1}{|x|}\right\}}\left|f_{\gamma}^{+}(t, s)\right|^{\xi} d s \nu(d x)<\infty
$$

and

$$
\int_{|x|<1}|x|^{\xi} \nu(d x)<\infty .
$$

For $\gamma<0$ we choose $\epsilon>0$ small enough such that for all $|x|<\epsilon$ the set $\{s \in \mathbb{R}$ : $\left.\left|f_{\gamma}^{+}(t, s)\right| \leq \frac{1}{|x|}\right\}$ is contained in $\mathbb{R} \backslash\left(\left\{s<0:|s|^{\gamma} \leq \frac{1}{|x|}\right\} \cup\left\{s<t:|t-s|^{\gamma} \leq \frac{1}{|x|}\right\}\right)$. Thus, we have by Proposition 2.2 that

$$
\int_{|x|<\epsilon}|x|^{\xi} \int_{\left\{s \in \mathbb{R}:\left|f_{\gamma}^{+}(t, s)\right| \leq \frac{1}{|x|}\right\}}\left|f_{\gamma}^{+}(t, s)\right|^{\xi} d s \nu(d x)<\infty
$$

if and only if

$$
\begin{aligned}
& \int_{|x|<\epsilon}|x|^{\xi}\left(K_{2}+K_{3} \int_{-1}^{-|x|^{-\frac{1}{\gamma}}}|s|^{\xi \gamma} d s\right) \nu(d x) \\
& =\int_{|x|<\epsilon}|x|^{\xi}\left(K_{4}+K_{5}|x|^{-\xi-\frac{1}{\gamma}}\right) \nu(d x)<\infty,
\end{aligned}
$$


where $K_{2}, K_{3}, K_{4}$ and $K_{5}$ denote constants. The last inequality is equivalent to

$$
\int_{|x|<1}|x|^{\xi \wedge\left(-\frac{1}{\gamma}\right)} \nu(d x)<\infty .
$$

This concludes the proof.

Suppose that for some $2 \geq \beta>0$ and $\eta>0$ the following two conditions hold

$$
\begin{aligned}
& \int_{|x|<1}|x|^{\beta} \nu(d x)<\infty, \\
& \int_{|x|>1}|x|^{\eta} \nu(d x)<\infty .
\end{aligned}
$$

Condition (8) means that the Lévy process $L$ with triplet $(0,0, \nu)$ possesses a finite $\eta$-th moment, whereas condition (7) is certainly satisfied if $\beta>\alpha$, where $\alpha$ denotes the Blumenthal-Getoor index of $L$, i.e.

$$
\inf \left\{u \geq 0: \int_{\mathbb{R}_{0}} 1 \wedge|x|^{u} \nu(d x)<\infty\right\} .
$$

As an application of the above proposition the following holds.

Corollary 1. Let $L$ be a Lévy process with characteristic triplet $(b, 0, \nu)$ whose Lévy measure $\nu$ satisfies (7) and (8). Then for any real number $\gamma$ with

$$
\gamma \in\left[-\frac{1}{\beta}, 1-\frac{1}{\eta}\right] \cap\left(-\infty, \frac{1}{2}\right)
$$

the first integrability condition (2) holds for $f_{\gamma}^{+}(t, \cdot)$ and any $t \in \mathbb{R}$, i.e.

$$
\int_{\mathbb{R}} \int_{\mathbb{R}_{0}}\left(\left|f_{\gamma}^{+}(t, s) x\right|^{2} \wedge 1\right) \nu(d x) d s<\infty .
$$

Proof. Setting $\delta=0$ and $\xi=2$, Proposition 3 directly yields the desired result.

Note that if we consider a symmetric pure jump Lévy process $L$, i.e. with characteristic triplet $(0,0, \nu)$, then the above condition is already sufficient for the integrability of $f_{\gamma}^{+}$. In fact, condition (3) holds trivially since the inner integral vanishes for any value of $f_{\gamma}^{+}(t, s)$. However, if $\nu$ is not symmetric, this condition imposes additional restrictions on the parameter $\gamma$.

Corollary 2. Let $L$ be a Lévy process with characteristic triplet $(b, 0, \nu)$ whose Lévy measure $\nu$ satisfies (7) and (8). Then for any real number $\gamma$ with

$$
\gamma \in\left[-\frac{1}{\beta}, 1-\frac{1}{\eta}\right] \cap(-1,0)
$$

both integrability conditions (2) and (3) hold for $f_{\gamma}^{+}(t, \cdot)$ and any $t \in \mathbb{R}$.

Proof. Since the assumptions of Corollary 1 are satisfied we only need to check integrability condition (3). This, however, clearly holds if

$$
\begin{aligned}
& \int_{\mathbb{R}}\left|f_{\gamma}^{+}(t, s)\left(b+\int_{\mathbb{R}_{0}} x\left(1_{\left\{\left|f_{\gamma}^{+}(t, s) x\right| \leq 1\right\}}-1_{\{|x| \leq 1\}}\right) \nu(d x)\right)\right| d s \\
& \leq \int_{\mathbb{R}}\left|f_{\gamma}^{+}(t, s) b\right| d s+\int_{\mathbb{R}} \int_{\mathbb{R}_{0}}\left|f_{\gamma}^{+}(t, s) x\left(1_{\left\{\left|f_{\gamma}^{+}(t, s) x\right| \leq 1\right\}}-1_{\{|x| \leq 1\}}\right)\right| \nu(d x) d s<\infty .
\end{aligned}
$$


By Proposition 2.3 the first summand is finite for any $b \in \mathbb{R}$, since $\gamma \in(-1,0)$. For the second summand it suffices to integrate on the sets $E_{1}=\left\{(x, s) \in \mathbb{R}_{0} \times \mathbb{R}:\left|f_{\gamma}^{+}(t, s) x\right|>1,|x| \leq 1\right\}$ and $E_{2}=\left\{(x, s) \in \mathbb{R}_{0} \times \mathbb{R}:\left|f_{\gamma}^{+}(t, s) x\right| \leq 1,|x|>1\right\}$ where the indicator functions do not cancel each other out. By the proof of Proposition 3 we have for all $\gamma$ with $\gamma>-1$ and $\gamma \geq-\frac{1}{\beta}$

$$
\int_{E_{1}}\left|f_{\gamma}^{+}(t, s) x\right| \lambda \otimes \nu(d s d x)<\infty
$$

Similarly, for all $\gamma$ with $\gamma<0$ and $\gamma \leq 1-\frac{1}{\eta}$ we obtain

$$
\int_{E_{2}}\left|f_{\gamma}^{+}(t, s) x\right| \lambda \otimes \nu(d s d x)<\infty
$$

This concludes the proof.

In the above situation we had to restrict the parameter $\gamma$ to the interval $(-1,0)$ which ensured the integrability of the kernel function at zero and infinity. It is, however, possible to allow for a broader range of values of $\gamma$. In these cases we have either to compensate the small jumps or the big jumps of $L$ which requires finite variation or finite first moments, respectively. Let us start with the former case.

Corollary 3. Let $L$ be a Lévy process with finite variation and characteristic triplet $(b, 0, \nu)$ whose Lévy measure $\nu$ satisfies (7) and (8) with $\beta \leq 1$. Furthermore, let the drift b be given by

$$
b=\int_{|x| \leq 1} x \nu(d x) .
$$

Then for any real number $\gamma$ with

$$
\gamma \in\left[-\frac{1}{\beta},\left(1-\frac{1}{\eta}\right) \wedge 0\right]
$$

both integrability conditions (2) and (3) hold for $f_{\gamma}^{+}(t, \cdot)$ and any $t \in \mathbb{R}$.

Proof. By Corollary 1 condition (2) clearly holds. With $b$ as above, the second condition (3) simplifies to

$$
\int_{\mathbb{R}}\left|\int_{\mathbb{R}_{0}} f_{\gamma}^{+}(t, s) x 1_{\left\{\left|f_{\gamma}^{+}(t, s) x\right| \leq 1\right\}} \nu(d x)\right| d s<\infty
$$

which holds by Prop. 3 especially if $\gamma<0$ and $\gamma \in\left[-\frac{1}{\beta}, 1-\frac{1}{\eta}\right]$.

In the case of a Lévy process with finite variation we do not have to restrict $\gamma$ to negative values.

Corollary 4. Let $L$ be a Lévy process with finite first moments and characteristic triplet $(b, 0, \nu)$ whose Lévy measure $\nu$ satisfies (7) and (8) with $\eta \geq 1$. Furthermore, let the drift $b$ be given by

$$
b=-\int_{|x|>1} x \nu(d x) .
$$

Then for any real number $\gamma$ with

$$
\gamma \in\left[-\frac{1}{\beta}, 1-\frac{1}{\eta}\right] \cap\left(-1, \frac{1}{2}\right)
$$

both integrability conditions (2) and (3) hold for $f_{\gamma}^{+}(t, \cdot)$ and any $t \in \mathbb{R}$. 
Proof. We first note that by Corollary 1 condition (2) is clearly satisfied. Moreover, in this situation, for any $t \in \mathbb{R}$, the second integrability condition (3) takes the form

$$
\int_{\mathbb{R}}\left|\int_{\mathbb{R}_{0}} f_{\gamma}^{+}(t, s) x 1_{\left\{\left|f_{\gamma}^{+}(t, s) x\right|>1\right\}} \nu(d x)\right| d s<\infty .
$$

By Proposition 3 with $\delta=1$ this holds especially if $\gamma>-1$ and $\gamma \in\left[-\frac{1}{\beta}, 1-\frac{1}{\eta}\right]$.

We are now able to define fractional Lévy processes.

Definition 1. Let $L$ and $\gamma$ be as in one of the three corollaries above. We define a stochastic process by

$$
X_{\gamma}^{+}(t)=\int_{\mathbb{R}} f_{\gamma}^{+}(t, s) L(d s), \quad t \in \mathbb{R},
$$

which we call a fractional Lévy process.

Note that the above results on the integrability remain true if we replace the function $f_{\gamma}^{+}$by either $f_{\gamma}^{-}$or $f_{\gamma}$. In fact, we observe that $f_{\gamma}^{-}(t, s)=-f_{\gamma}^{+}(t, t-s)$ and $f_{\gamma}(t, s)=f_{\gamma}^{+}(t, s)+f_{\gamma}^{-}(t, s)$ for all $t, s \in \mathbb{R}$. Now it suffices to recall that $L_{\phi_{0}}(\mathbb{R})$ is a linear space. Thus, we can as well define fractional Lévy processes by integrating these kernel functions or any of their linear combinations $a f_{\gamma}^{+}+b f_{\gamma}^{-}, a, b \in \mathbb{R}$. The properties of the integrals which we will analyze in the following hold for any of these functions. However, for the sake of simplicity we will formulate the results for $f_{\gamma}^{+}$.

Proposition 4. Let $L$ and $\gamma$ be as in the above definition. In particular, $L$ has characteristic triplet $(b, 0, \nu)$ with $b \in \mathbb{R}, b=\int_{|x| \leq 1} x \nu(d x)$ or $b=-\int_{|x|>1} x \nu(d x)$, respectively.

Then the process $X_{\gamma}^{+}$in Definition 1 has stationary increments. Moreover, for $n \in \mathbb{N}, t_{1}, \ldots, t_{n} \in$ $\mathbb{R}$ and $u_{1}, \ldots, u_{n} \in \mathbb{R}$ its finite dimensional distributions have characteristic function given by

$$
E\left[\exp \left\{\mathrm{i} \sum_{i=1}^{n} u_{i} X_{\gamma}^{+}\left(t_{i}\right)\right\}\right]=\exp \left\{\int_{\mathbb{R}} \psi_{\gamma}\left(\sum_{i=1}^{n} u_{i} f_{\gamma}^{+}\left(t_{i}, s\right)\right) d s\right\},
$$

where

$$
\psi_{\gamma}(y)=\mathrm{i} y b+\int_{\mathbb{R}_{0}}\left(e^{\mathrm{i} y x}-1-\mathrm{i} y x 1_{\{|x| \leq 1\}}\right) \nu(d x), \quad y \in \mathbb{R} .
$$

In particular, $X_{\gamma}^{+}(t)$ is infinitely divisible for all $t \in \mathbb{R}$ with characteristic triplet $\left(b_{f_{\gamma}^{+}}, 0, \nu_{f_{\gamma}^{+}}\right)$ given by

$$
\begin{array}{rlrl}
b_{f_{\gamma}^{+}} & =\int_{\mathbb{R}_{\gamma}} f_{\gamma}^{+}(t, s)\left(b+\int_{\mathbb{R}_{0}} x\left(1_{\left\{\left|f_{\gamma}^{+}(t, s) x\right| \leq 1\right\}}-1_{\{|x| \leq 1\}}\right) \nu(d x)\right) d s, & & \\
\nu_{f_{\gamma}^{+}}(B) & =\int_{\mathbb{R}} \int_{\mathbb{R}_{0}} 1_{B}\left(f_{\gamma}^{+}(t, s) x\right) \nu(d x) d s, & B \in \mathcal{B}\left(\mathbb{R}_{0}\right) .
\end{array}
$$

Proof. The form of the characteristic function is a direct consequence of Proposition 2.4 in Rajput and Rosiński (1989) if we note that $\sum_{i=1}^{n} u_{i} f_{\gamma}^{+}\left(t_{i}, \cdot\right)$ is integrable since $L_{\phi_{0}}(\mathbb{R})$ is a linear space (see Prop. 1). Theorem 2.7 in the same paper yields the representation of the characteristic triplet. The stationarity of increments is a consequence of the same property of the Lévy process. 
Example 1. An important example of fractional Lévy processes are the so-called linear fractional stable motions which were introduced in Samorodnitsky and Taqqu (1994, Example 3.6.5 and Section 7.4) as

$$
Y_{H}^{\alpha}(t)=\int_{\mathbb{R}}(t-s)_{+}^{H-1 / \alpha}-(-s)_{+}^{H-1 / \alpha} M(d s), \quad t \in \mathbb{R},
$$

where $M$ is an $\alpha$-stable random measure. Here $0<\alpha<2$ is the stability index and $H \in(0,1)$. These processes arise as a special case of our definition of fractional Lévy processes. For instance, in the symmetric case, define a Lévy measure by $\nu_{\alpha}(d x)=c|x|^{-1-\alpha} d x$. Clearly, (7) holds for any $\beta \in(\alpha, \infty)$ and (8) holds for any $\eta \in[0, \alpha)$. By choosing the Lévy process $L_{\alpha}$ with Lévy measure $\nu_{\alpha}$ as in Corollary 2, 3 or 4 for $\alpha=1, \alpha \in(0,1)$ or $\alpha \in(1,2)$, respectively, we can define the fractional Lévy process

$$
X_{\gamma}^{\alpha}(t)=\int_{\mathbb{R}} f_{\gamma}^{+}(t, s) L_{\alpha}(d s), \quad t \in \mathbb{R},
$$

for $\gamma \in\left(-\frac{1}{\alpha}, 1-\frac{1}{\alpha}\right)$. Using the characteristic functions it is easy to see that, for $\gamma=H-1 / \alpha$, $Y_{H}^{\alpha}$ and $X_{\gamma}^{\alpha}$ are equal in distribution.

\section{Distributional properties}

Using Proposition 3 it is now easy to give sufficient conditions for the existence of moments of the integrated process.

Proposition 5. Let $L$ be as in the previous proposition and $p \geq 0$. If

$$
\left.p \leq \eta \text { and } p<\frac{1}{-\gamma} \text { for } \gamma<0\right)
$$

then for any $t \in \mathbb{R}$ the $p$-th moment of $X_{\gamma}^{+}(t)$ exists, i.e. $E\left|X_{\gamma}^{+}(t)\right|^{p}<\infty$.

Proof. We first recall that the existence of $p$-th moments of $L$ is equivalent to

$$
\int_{|x|>1}|x|^{p} \nu(d x)<\infty .
$$

By Prop. 1 it suffices to show that for any $t \in \mathbb{R}$

$$
\int_{\mathbb{R}} \int_{\mathbb{R}_{0}} 1_{\left\{\left|f_{\gamma}^{+}(t, s) x\right|>1\right\}}\left|f_{\gamma}^{+}(t, s) x\right|^{p} \nu(d x) d s<\infty
$$

However, $p \leq \eta$ and $\gamma>-\frac{1}{p}$ together with Prop. 3 yield the desired result.

The above proposition provides sufficient conditions for the existence of the $p$-th moments of $X_{\gamma}^{+}$. If $p$ is a positive integer, Proposition 4 enables us to compute the $p$-th moment explicitly. Let us consider the important case $p=2$, then we need that $L$ is square-integrable, i.e., $\eta \geq 2$. Since in this case, $L$ clearly has finite first moments, the assumptions of Corollary 4 hold and we choose $b=-\int_{|x|>1} x \nu(d x)$. This corollary and Proposition 5 show that $X_{\gamma}^{+}$is square-integrable for any $\gamma \in\left(-\frac{1}{2}, \frac{1}{2}\right)$. By Theorem 15.31 in Klenke (2006) we are now able to compute the first 
and the second moments of $X_{\gamma}^{+}$. This yields for any $t \in \mathbb{R}$

$$
\begin{aligned}
E X_{\gamma}^{+}(t) & =-\left.i \frac{\partial}{\partial u} E \exp \left(i u X_{\gamma}^{+}(t)\right)\right|_{u=0} \\
& =-\left.i \frac{\partial}{\partial u} \exp \left\{\int_{\mathbb{R}} \int_{\mathbb{R}_{0}}\left(e^{\mathrm{i} u f_{\gamma}^{+}(t, s) x}-1-\mathrm{i} u f_{\gamma}^{+}(t, s) x\right) \nu(d x) d s\right\}\right|_{u=0} \\
& =0
\end{aligned}
$$

where by dominated convergence we can interchange differentiation and integration. By similar arguments the second moment is given by

$$
\begin{aligned}
E\left(X_{\gamma}^{+}(t)\right)^{2} & =-\left.\frac{\partial^{2}}{\partial u^{2}} E \exp \left(i u X_{\gamma}^{+}(t)\right)\right|_{u=0} \\
& =\int_{\mathbb{R}_{0}} x^{2} \nu(d x) \int_{\mathbb{R}}\left(f_{\gamma}^{+}(t, s)\right)^{2} d s \\
& =t^{2 \gamma+1} E(L(t))^{2} \int_{\mathbb{R}}\left(f_{\gamma}^{+}(1, s)\right)^{2} d s .
\end{aligned}
$$

Example 2. The above situation of a square-integrable Lévy process has been considered by Benassi et al. (2004) and Marquardt (2006). Their processes can be recovered from our results by setting $b=-\int_{|x|>1} x \nu(d x)$ and $\gamma=H-1 / 2$ for $H \in(0,1)$. As seen above, the arising process

$$
X_{H}^{+}(t)=\int_{\mathbb{R}}(t-s)_{+}^{H-1 / 2}-(-s)_{+}^{H-1 / 2} L(d s), \quad t \in \mathbb{R}
$$

is well-defined and square-integrable. Further properties are studied in Marquardt (2006) for the case $H-1 / 2>0$ where the process $X_{H}^{+}$exhibits long range dependence.

Remark 1. Note that if $X_{\gamma}^{+}$is square-integrable with variance given by (14), then it essentially has the same correlation structure as fractional Brownian motion, namely

$$
\operatorname{Cov}\left(X_{\gamma}^{+}(t), X_{\gamma}^{+}(u)\right)=E(L(t))^{2} \int_{\mathbb{R}}\left(f_{\gamma}^{+}(1, s)\right)^{2} d s\left(|t|^{2 \gamma+1}+|u|^{2 \gamma+1}-|t-u|^{2 \gamma+1}\right) .
$$

Therefore, $X_{\gamma}^{+}$exhibits short range dependence if $\gamma<0$ and long range dependence if $\gamma>$ 0 . Hence, to be in line with the structure of fractional Brownian motion we could use the parametrisation $\gamma=H-1 / 2$ with $H \in(0,1)$ as it was done in Marquardt (2006).

\section{Path properties}

Path properties of fractional Lévy processes under the condition of vanishing first moments and finite second moments have been proved in Marquardt (2006) and Benassi et al. (2004). They showed that for $\gamma<0$ the sample paths are unbounded on any interval since the kernel in this case is unbounded. This argument also holds in our more general setting.

For $\gamma>0$ they showed that the process possesses a Hölder continuous version of the order $d$ with $d<\gamma$. We will show that this also holds true in our more general setting, namely when $\beta<\eta$. However, we have to use a different technique to prove this since we cannot use the moment structure derived by the characteristic function. Instead we have to go via the improper Riemann integral representation and the short time behaviour of absolute moments of a Lévy process. 
Proposition 6. Let $L$ be a Lévy process with finite first moments and characteristic triplet $(b, 0, \nu)$ whose Lévy measure $\nu$ satisfies (7) and (8) with $\eta>1$. Furthermore, let the drift $b$ be given by

$$
b=-\int_{|x|>1} x \nu(d x) .
$$

Then for any real number $\gamma$ with

$$
\gamma \in\left[-\frac{1}{\beta}, 1-\frac{1}{\eta}\right) \cap\left(0, \frac{1}{2}\right)
$$

$X_{\gamma}^{+}$possesses a continuous version

$$
X_{\gamma}^{+}(t)=\int \gamma f_{\gamma-1}^{+}(t, s) L(s) d s
$$

If $\beta<\eta$, it is also Hölder continuous of the order $d$ with $d<\gamma$.

Proof. It is clear that we have to restrict ourselves to positive $\gamma$, since otherwise the kernel function is unbounded and hence also the associated process. Then the representation of $X_{\gamma}^{+}$ as improper Riemann integral follows straight forward by integration by parts as in Marquardt (2006). We only have to note that since we do not assume a finite second moment we cannot use the law of iterated logarithm to show that

$$
\lim _{s \rightarrow-\infty} L(s)\left((t-s)^{\gamma}-(-s)^{\gamma}\right)=0 \quad \text { a.s. }
$$

However instead we can use Proposition 48.10 of Sato (1999), which yields

$$
\limsup _{t \rightarrow \infty} \frac{\sup _{0 \leq s \leq t}|L(s)|}{t^{1 / r}}=0 \quad \text { a.s. }
$$

for $r<\bar{\beta}$. By Pruitt (1981) we know that if $E|L(t)|<\infty$ and $E L(t) \neq 0$ then $\bar{\beta}=1$, and otherwise $\bar{\beta}=\sup \left\{\gamma \in[0,2]: \int_{|x|>1}|x|^{\gamma} \nu(d x)<\infty\right\}$.

Continuity of $X_{\gamma}^{+}$follows as in Marquardt (2006). For Hölder continuity the arguments are different. First note that by a change of variables we obtain

$$
X_{\gamma}^{+}(t)=\gamma t^{\gamma} \int f_{\gamma-1}^{+}(1, u) L(u t) d u
$$

Now we use that by Woerner (2003) $E|L(t)|^{r} \leq t C$ for $t \rightarrow 0$ if $\eta \geq r>\beta$ and obtain by Minkowski's inequality that for $t \rightarrow 0$

$$
\begin{aligned}
\left(E\left|X_{\gamma}^{+}(t)\right|^{\eta}\right)^{1 / \eta} & =\gamma t^{\gamma}\left(E\left|\int f_{\gamma-1}^{+}(1, u) L(t u) d u\right|^{\eta}\right)^{1 / \eta} \\
& \leq \gamma t^{\gamma} \int\left|f_{\gamma-1}^{+}(1, u)\right|\left(E|L(u t)|^{\eta}\right)^{1 / \eta} d u \\
& \leq t^{\gamma+\frac{1}{\eta}} \gamma C \int\left|f_{\gamma-1}^{+}(1, u)\right||u|^{1 / \eta} d u<\infty,
\end{aligned}
$$

where the last integral is finite since $\gamma<1-\frac{1}{\eta}$. Hence we obtain as $t \rightarrow 0$

$$
E\left|X_{\gamma}^{+}(t)\right|^{\eta} \leq t^{\eta \gamma+1} C .
$$

Using Kolmogorov-Centsov we now obtain Hölder continuity of the order $d$ with $d<\gamma$. 
Note that this proposition does not hold for stable processes, since for them we have $\eta<\beta$. However, here we can use that by Example 25.10 of Sato (1999) for a symmetric $\alpha$-stable process, $\alpha \in(1,2)$ we have $E|L(t)|^{p}=t^{p / \alpha} C$ for $-1<p<\alpha$. Hence by inserting this is the calculation above we obtain $E\left|X_{\gamma}^{+}(t)\right|^{p} \leq t^{p(\gamma+1 / \alpha)}$ for $p<\alpha$, which also yields Hölder continuity of the order $d$ with $d<\gamma$.

By the previous result we see that in contrast to fractional Brownian motion we only have continuous sample paths in the long-range dependent case and the sample paths are less regular, namely at most Hölder continuous of the order less than $1-1 / \eta \leq 1 / 2$.

Proposition 7. Let $L$ be a Lévy process with finite first moments and characteristic triplet $(b, 0, \nu)$ whose Lévy measure $\nu$ satisfies (7) and (8) with $\eta>1$. Furthermore, let the drift $b$ be given by

$$
b=-\int_{|x|>1} x \nu(d x)
$$

Then it is equivalent that

(a)

$$
\gamma \in\left[1-\frac{1}{\beta}, 1-\frac{1}{\eta}\right) \cap\left(0, \frac{1}{2}\right)
$$

with $\beta$ chosen to be the minimal one and $\eta$ the maximal one which is smaller or equal to two, and

(b) $X_{+}^{\gamma}$ is a semimartingale of bounded variation with respect to any filtration it is adapted to.

Proof. The proof is analogous to the proof in Bender et al. (2010). Though they worked under the condition of finite second moments, they mainly used it to obtain an appropriate decay of the Lévy process by a law of iterated logarithm. Instead of this we can, as in the previous proof use (16). Furthermore, they used the property of finite second moments in Lemma 3.5. to show that for $r, t>0$ as $t \rightarrow 0$

$$
E\left(\left|\frac{1}{t} \int_{-\infty}^{t}\left((t-s)_{+}^{\gamma}-(-s)_{+}^{\gamma}\right) L(d s)-\gamma \int_{-\infty}^{0}(-s)^{\gamma-1} L(d s)\right|\right) \rightarrow 0 .
$$

To show this we can use the same arguments as in the previous proof. First we write the expression as improper Riemann-integral, where the boundary terms vanish by the same argument as before. We obtain

$$
\begin{aligned}
Y(t) & =\frac{1}{t} \int_{-\infty}^{t}\left((t-s)_{+}^{\gamma}-(-s)_{+}^{\gamma}\right) L(d s)-\gamma \int_{-\infty}^{0}(-s)^{\gamma-1} L(d s) \\
& =\gamma \int \frac{1}{t}\left((t-s)_{+}^{\gamma-1}-(-s)_{+}^{\gamma-1}-(\gamma-1) t(-s)_{+}^{\gamma-2}\right) L(s) d s \\
& =t^{\gamma-1} \gamma \int\left((1-u)_{+}^{\gamma-1}-(-u)_{+}^{\gamma-1}-(\gamma-1)(-u)_{+}^{\gamma-2}\right) L(u t) d u
\end{aligned}
$$

Note that we indeed have $\beta<\eta$ and $\eta>1$, since otherwise the range of $\gamma$ is empty. Hence by the same argument as in the previous proof we obtain as $t \rightarrow 0$ and $\beta \leq 1<p<\eta$

$$
E|Y(t)| \leq\left(E|Y(t)|^{p}\right)^{1 / p} \leq t^{\gamma-1+1 / p} C<\infty
$$

which tends to zero since $\gamma>1-1 / p$. This completes the proof. 
Note that obviously linear fractional stable motion and fractional Brownian motion cannot be semimartingales since in this case we have $\beta=\eta$ and hence the range of $\gamma$ is empty.

Since under the conditions of the previous proposition the fractional Lévy process is a semimartingale of finite variation, it has the semimartingale characteristics $\left(B_{t}, 0,0\right)$, hence it is a generalized drift $B_{t}$. This implies that semimartingale fractional Lévy processes might serve as drift component to introduce long-range dependence to a Brownian semimartingale model, e.g. a classical stochastic volatility model.

For the case $\gamma<1-1 / \beta$ we restrict ourselves to the class of driving Lévy processes whose Lévy measure locally around zero behaves like the one of a stable process. The following proposition is a generalization of Theorem 4.5 in Marquardt (2006) and the proof is essentially the same and will therefore be omitted.

Proposition 8. Let $\alpha \in(0,2)$ and suppose that the Lévy measure $\nu$ has a Lebesgue density $g$, such that

$$
\begin{aligned}
& \lim _{x \rightarrow 0} \frac{g(x)}{|x|^{-1-\alpha}}=c, \\
& g(x) \leq C|x|^{-1-\alpha}, \quad \text { for all } x \in \mathbb{R},
\end{aligned}
$$

where $c, C>0$, and an appropriate drift $b$ (depending on $\alpha$, cf. Example 1). For $\gamma \in$ $\left(-\frac{1}{\alpha}, 1-\frac{1}{\alpha}\right), X_{\gamma}^{+}$is locally self-similar with parameter $\gamma+1 / \alpha$. More precisely, for every fixed $t \in \mathbb{R}$, we have

$$
\lim _{\epsilon \downarrow 0}\left\{\frac{X_{\gamma}^{+}(t+\epsilon x)-X_{\gamma}^{+}(t)}{\epsilon^{\gamma+1 / \alpha}}\right\}_{x \in \mathbb{R}} \stackrel{(\mathrm{d})}{=}\left\{X_{\gamma}^{\alpha}(x)\right\}_{x \in \mathbb{R}},
$$

where the limit is in distribution for all finite-dimensional margins and $X_{\gamma}^{\alpha}$ is a linear fractional stable motion with parameters $\alpha$ and $c$ as in Example 1.

The above proposition tells us how the increments of the integrated process behave at small scale. The conditions are satisfied by popular classes of Lévy processes with infinite activity whose Lévy measures possess a Lebesgue density, e.g. CGMY processes or generalized hyperbolic Lévy processes.

Note that for fractional Brownian motion we have self similarity and hence local self-similarity with parameter $H$. Hence to be in line with this we would use the parameterization $\gamma=H-1 / \alpha$, $H \in(0,1)$ as for linear fractional stable motion.

The knowledge of the small time scaling can be used to show a result on the strong $p$-variation of the process $X_{\gamma}^{+}$.

Recall that the strong $p$-variation of a stochastic process $X$ on the interval $[a, b]$ is a measure of roughness of its sample paths which is defined as

$$
\operatorname{Var}_{[a, b]}^{p}(X)=\sup _{\tau}\left(\sum_{i=0}^{n-1}\left|X\left(t_{i+1}\right)-X\left(t_{i}\right)\right|^{p}\right)^{1 / p}
$$

where $p>0$ and the supremum is taken over all subdivisions $\tau$ of $[a, b]$ :

$$
\tau: a=t_{0}<t_{1}<\ldots<t_{n}=b, \quad n \geq 1 .
$$

Proposition 9. Let $\alpha \in(0,2)$ and suppose that the Lévy process $L$ is as in Proposition 8. Then for $\gamma \in\left(-\frac{1}{\alpha}, 1-\frac{1}{\alpha}\right)$, the process $X_{\gamma}^{+}$has a.s. infinite $p$-variation on every compact interval for all $0<p<\frac{1}{\gamma+1 / \alpha}$. In particular, the total variation is always infinite. 
Proof. Let $p<\frac{1}{\gamma+1 / \alpha}$. From Prop. 8 we conclude that for every $t \in \mathbb{R}$ we have

$$
\lim _{h \downarrow 0} \frac{\left|X_{\gamma}^{+}(t \pm h)-X_{\gamma}^{+}(t)\right|^{p}}{|h|^{p(\gamma+1 / \alpha)}} \stackrel{(\mathrm{d})}{=}\left|X_{\gamma}^{\alpha}( \pm 1)\right|^{p}>0, \quad \text { a.s. }
$$

Let $(\Omega, \mathcal{A}, \mathbb{P})$ be the underlying probability space and $\Omega^{\prime} \subset \Omega$ an arbitrary subset such that $\mathbb{P}\left(\Omega^{\prime}\right)>0$. Then, by the same arguments as in the proof of Theorem 4.6 in Marquardt (2006), we conclude

$$
\lim _{h \downarrow 0} E\left[1_{\Omega^{\prime}} \frac{\left|X_{\gamma}^{+}(t \pm h)-X_{\gamma}^{+}(t)\right|^{p}}{|h|^{p(\gamma+1 / \alpha)}}\right]>0 .
$$

Assume

$$
\mathbb{P}\left[\operatorname{Var}_{[a, b]}^{p}\left(X_{\gamma}^{+}\right)<\infty\right]=\mathbb{P}\left[\left(\operatorname{Var}_{[a, b]}^{p}\left(X_{\gamma}^{+}\right)\right)^{p}<\infty\right]>0
$$

Then we find an $\Omega^{\prime} \subset \Omega$ with $\mathbb{P}\left(\Omega^{\prime}\right)>0$ and a $K>0$ such that $\left(\operatorname{Var}_{[a, b]}^{p}\left(X_{\gamma}^{+}\right)\right)^{p}<K$ on $\Omega^{\prime}$ and thus

On the other hand, by (18) we observe

$$
E\left[1_{\Omega^{\prime}}\left(\operatorname{Var}_{[a, b]}^{p}\left(X_{\gamma}^{+}\right)\right)^{p}\right] \leq K
$$

$$
\lim _{h \downarrow 0} E\left[1_{\Omega^{\prime}} \frac{\left|X_{\gamma}^{+}(t \pm h)-X_{\gamma}^{+}(t)\right|^{p}}{h}\right]=\lim _{h \downarrow 0} h^{p(\gamma+1 / \alpha)-1} E\left[1_{\Omega^{\prime}} \frac{\left|X_{\gamma}^{+}(t \pm h)-X_{\gamma}^{+}(t)\right|^{p}}{h^{p(\gamma+1 / \alpha)}}\right]=\infty
$$

since $p(\gamma+1 / \alpha)-1<0$.

Now fix $b^{\prime} \in \mathbb{R}$ with $a<b^{\prime} \leq b$. By the above consideration we can find for every $t \in\left[a, b^{\prime}\right]$ an $\epsilon_{t}>0$ with $\epsilon_{t}<b-b^{\prime}$ such that for all $|h| \leq \epsilon_{t}$

$$
E\left[1_{\Omega^{\prime}}\left|X_{\gamma}^{+}(t \pm h)-X_{\gamma}^{+}(t)\right|^{p}\right] \geq \frac{2 K}{b^{\prime}-a}|h|
$$

holds. Since $\left\{\left(t-\epsilon_{t}, t+\epsilon_{t}\right)\right\}_{t \in\left[a, b^{\prime}\right]}$ is an open cover of the compact interval $\left[a, b^{\prime}\right]$ we obtain a finite subcover $\left\{\left(t_{2 i}-\epsilon_{t_{2 i}}, t_{2 i}+\epsilon_{t_{2 i}}\right)\right\}_{i=1}^{m}$. Furthermore, for $i \in\{1, \ldots, m-1\}$ we pick

$$
t_{2 i+1} \in\left(t_{2 i}, t_{2 i}+\epsilon_{t_{2 i}}\right) \cap\left(t_{2 i+2}-\epsilon_{t_{2 i+2}}, t_{2 i+2}\right)
$$

and define $t_{1}=a$ and $b^{\prime}<t_{2 m+1}=t_{2 m}+\epsilon_{t_{2 m}}<b^{\prime}$. It is now enough to observe that

$$
\begin{aligned}
E\left[1_{\Omega^{\prime}}\left(\operatorname{Var}_{[a, b]}^{p}\left(X_{\gamma}^{+}\right)\right)^{p}\right] & \geq \sum_{i=1}^{2 m} E\left[1_{\Omega^{\prime}}\left|X_{\gamma}^{+}\left(t_{i+1}\right)-X_{\gamma}^{+}\left(t_{i}\right)\right|^{p}\right] \\
& \geq \frac{2 K}{b^{\prime}-a} \sum_{i=1}^{2 m}\left|t_{i+1}-t_{i}\right|=\left(t_{2 m+1}-a\right) \frac{2 K}{b^{\prime}-a} \geq 2 K
\end{aligned}
$$

which contradicts (19). Hence,

$$
\mathbb{P}\left[\operatorname{Var}_{[a, b]}^{p}\left(X_{\gamma}^{+}\right)=\infty\right]=1 .
$$

Finally, from $1<\frac{1}{\gamma+1 / \alpha}$ for any $\gamma \in\left(-\frac{1}{\alpha}, 1-\frac{1}{\alpha}\right)$ we conclude

$$
\mathbb{P}\left[\operatorname{Var}_{[a, b]}^{1}\left(X_{\gamma}^{+}\right)=\infty\right]=1 \text {. }
$$


This result is similar to the case of fractional Brownian motion, where we also obtain infinite $p$-variation for $p$ less than one over the self-similarity index.

Summarizing we see that these differences between fractional Brownian motion and fractional Lévy processes imply that especially statistical inference by non-parametric methods on the degree of long-range dependence needs a thorough analysis when it is not clear of which class the true underlying process is. For example, relying on the Hölder continuity as measure of the dependence, this might suggest short range dependence instead of long range dependence, when the true process is a fractional Lévy process instead of a fractional Brownian motion.

Acknowledgements. The financial support of the Deutsche Telekom Stiftung (first author) and the Deutsche Forschungsgemeinschaft (SFB 823: Statistical modelling of nonlinear dynamic processes) (second author) is gratefully acknowledged.

\section{References}

A. Basse and J. Pedersen. Lévy driven moving averages and semimartingales. Stochastic Process. Appl., 119(9):2970-2991, 2009.

A. Benassi, S. Cohen, and J. Istas. On roughness indices for fractional fields. Bernoulli, 10(2): $357-373,2004$.

C. Bender, A. Lindner, and M. Schicks. Finite variation of fractional Lévy processes. Submitted, http://www.tu-braunschweig.de/Medien-DB/stochastik/bender-lindner-schicks1.pdf, 2010.

A. Klenke. Wahrscheinlichkeitstheorie. Springer-Verlag, Berlin, 2006.

B. B. Mandelbrot and J. W. Van Ness. Fractional Brownian motions, fractional noises and applications. SIAM Rev., 10:422-437, 1968.

T. Marquardt. Fractional Lévy processes with an application to long memory moving average processes. Bernoulli, 12(6):1099-1126, 2006.

J. Musielak. Orlicz spaces and modular spaces, volume 1034 of Lecture Notes in Mathematics. Springer-Verlag, Berlin, 1983.

W. E. Pruitt. The growth of random walks and Lévy processes. Ann. Probab., 9(6):948-956, 1981.

B. S. Rajput and J. Rosiński. Spectral representations of infinitely divisible processes. Probab. Theory Related Fields, 82(3):451-487, 1989.

G. Samorodnitsky and M. S. Taqqu. Stable non-Gaussian random processes. Stochastic Modeling. Chapman \& Hall, New York, 1994. Stochastic models with infinite variance.

K. Sato. Lévy processes and infinitely divisible distributions, volume 68 of Cambridge Studies in Advanced Mathematics. Cambridge University Press, Cambridge, 1999.

K. Urbanik and W. A. Woyczynski. Random integrals and orlicz spaces. Bull. Acad. Polon. Sci., 15:161-169, 1967.

J. H. C. Woerner. Variational sums and power variation: a unifying approach to model selection and estimation in semimartingale models. Statist. Decisions, 21(1):47-68, 2003. 


\section{Preprints ab 2008/25}

2010-16

2010-15

2010-14

2010-13

2010-12

2010-11

2010-10

2010-09

2010-08

2010-07

2010-06

2010-05

2010-04

2010-03

2010-02

2010-01

2009-16

2009-15

Sebastian Engelke and Jeannette H.C. Woerner

A unifying approach to fractional Lévy processes

Alexander Schnurr and Jeannette H.C. Woerner

Well-balanced Lévy Driven Ornstein-Uhlenbeck Processes

Lorenz J. Schwachhöfer

On the Solvability of the Transvection group of Extrinsic

Symplectic Symmetric Spaces

Marco Veneroni

Stochastic homogenization of subdifferential inclusions via scale integration

Agnes Lamacz, Andreas Rätz, and Ben Schweizer

A well-posed hysteresis model for flows in porous media and

applications to fingering effects

Luca Lussardi and Annibale Magni

$\Gamma$-limits of convolution functionals

Patrick W. Dondl, Luca Mugnai, and Matthias Röger

Confined elastic curves

Matthias Röger and Hendrik Weber

Tightness for a stochastic Allen-Cahn equation

Michael Voit

Multidimensional Heisenberg convolutions and product formulas

for multivariate Laguerre polynomials

Ben Schweizer

Instability of gravity wetting fronts for Richards equations with hysteresis

Lorenz J. Schwachhöfer

Holonomy Groups and Algebras

Agnes Lamacz

Dispersive effective models for waves in heterogeneous media

Ben Schweizer and Marco Veneroni

Periodic homogenization of Prandtl-Reuss plasticity equations in arbitrary dimension

Holger Dette and Karl Friedrich Siburg and Pavel A. Stoimenov

A copula-based nonparametric measure of regression dependence

René L. Schilling and Alexander Schnurr

The Symbol Associated with the Solution of a Stochastic Differential Equation

Henryk Zähle

Rates of almost sure convergence of plug-in estimates for distortion risk measures

Lorenz J. Schwachhöfer

Nonnegative curvature on disk bundles

Iuliu Pop and Ben Schweizer

Regularization schemes for degenerate Richards equations and outflow conditions 
Tom Krantz, Lorenz J. Schwachhöfer

Extrinsically Immersed Symplectic Symmetric Spaces

2009-12 Alexander Kaplun

Continuous time Ehrenfest process in term structure modelling

2009-11 Henryk Zähle

Ein aktuarielles Modell für die Portabilität der Alterungsrückstellungen in der PKV

2009-10 Andreas Neuenkirch and Henryk Zähle

Asymptotic error distribution of the Euler method for SDEs with

non-Lipschitz coefficients

2009-09 Karl Friedrich Siburg, Pavel A. Stoimenov

Regression dependence

2009-08 Wilfried Hazod

Continuous convolution hemigroups integrating a sub-multiplicative function

2009-07 Sergio Conti and Ben Schweizer

On optimal metrics preventing mass transfer

2009-06 Simon Castle, Norbert Peyerimhoff, Karl Friedrich Siburg

Billiards in ideal hyperbolic polygons

2009-05 Ludwig Danzer

Quasiperiodic Tilings - Substitution Versus Inflation

2009-04 Flavius Guiaş

Direct simulation of the infinitesimal dynamics of semi-discrete approximations for convection-diffusion-reaction problems

2009-03 Franz Kalhoff and Victor Pambuccian

Existential definability of parallelism in terms of betweenness

in Archimedean ordered affine geometry

2009-02 Fulvia Buzzi, Michael Lenzinger and Ben Schweizer

Interface conditions for degenerate two-phase flow equations in one space dimension

2009-01 Henryk Zähle

Approximation of SDEs by population-size-dependent

Galton-Watson processes

2008-25 Wilfried Hazod

Mehler semigroups, Ornstein-Uhlenbeck processes and background driving Lévy processes on locally compact groups and on hypergroups 